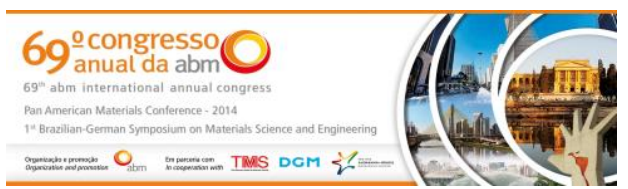

Tema: Produtos metálicos-não ferrosos

\title{
PROPRIEDADES MECÂNICAS E RECRISTALIZAÇÃO ESTÁTICA DA LIGA DE MAGNÉSIO ZK60A CONFORMADA A FRIO POR TREFILAÇÃO*
}

\author{
Diogo de Oliveira Azevedo ${ }^{1}$ \\ Klaus Higor dos Santos Silva² \\ Gilmar Cordeiro Silva ${ }^{3}$
} Pedro Brito 4

\section{Resumo}

No presente trabalho, processos de conformação a frio e recozimento foram aplicados à liga de magnésio ZK60A (Mg-6\%Zn-1\%Zr) com o objetivo de determinar a cinética dos fenômenos de recuperação e recristalização para permitir o refino de grão. Para tanto, foi desenvolvida uma série de matrizes de conformação para a trefilação incremental de barras circulares da liga ZK60A inicialmente recebida no estado envelhecido (T5), resultando em reduções de 7,8\%, 15,4\%, 22,6\% e 29,4\%. Após cada passe de trefilação, as propriedades mecânicas do material foram determinadas. $O$ estudo do recozimento foi realizado em amostras com o máximo encruamento a temperaturas de $400^{\circ} \mathrm{C}, 350^{\circ} \mathrm{C}, 300^{\circ} \mathrm{C}, 250^{\circ} \mathrm{C}, 200^{\circ} \mathrm{C}$ e $150^{\circ} \mathrm{C}$. Pela metodologia aplicada, foi possível avaliar a cinética do processo de recristalização da liga ZK60A e determinar a sua energia de ativação.

Palavras-chave: Liga de magnésio; Conformação; Recozimento; Energia de ativação.

\section{MECHANICAL PROPERTIES AND STATIC RECRYSTALLIZATION OF COLD- DRAWN ZK60A MAGNESIUM ALLOY}

\section{Abstract}

In the present study a combination of cold drawing processes followed by annealing treatments was applied to a ZK60A alloy (Mg-6\%Zn-1\%Zr) with the objective of investigating the conditions, which allowed grain size refinement. Forming dies were developed to allow the incremental drawing of ZK60A round bars (received in the T5 condition). The bars were cold worked to a $7.8 \%, 15.4 \%, 22.6 \%$ and $29.4 \%$ reduction ratio. After each deformation step, tensile properties and hardness were evaluated. The recrystallization process was investigated by submitting the $29.4 \%$ cold worked samples to annealing treatments at $400^{\circ} \mathrm{C}, 350^{\circ} \mathrm{C}, 300^{\circ} \mathrm{C}, 250^{\circ} \mathrm{C}, 200^{\circ} \mathrm{C}$ and $150^{\circ} \mathrm{C}$. The microstructure of strain-hardened and annealed specimens was characterized by optical microscopy. Finally, the activation energy for the recrystallization process of the cold drawn ZK60A alloy could be determined.

Keywords: Magnesium alloy; Cold-forming; Annealing; Activation energy.

1 Estudante de Engenharia Mecânica, Departamento de Engenharia Mecânica, Pontifícia Universidade Católica de Minas Gerais, Belo Horizonte, MG, Brasil.

2 Estudante de Pós-Graduação em Engenharia Mecânica, Departamento de Engenharia Mecânica, Pontifícia Universidade Católica de Minas Gerais, Belo Horizonte, MG, Brasil.

3 Dr. Msc. Engenheiro Mecânico, Professor Adjunto IV, Departamento de Engenharia Mecânica, Pontifícia Universidade Católica de Minas Gerais, MG, Brasil.

4 Dr.-Ing. Msc. Engenheiro Mecânico, Professor Adjunto IV, Departamento de Engenharia Mecânica, Pontifícia Universidade Católica de Minas Gerais, MG, Brasil.

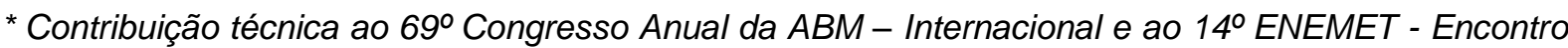
Nacional de Estudantes de Engenharia Metalúrgica, de Materiais e de Minas, 21 a 25 de julho de 2014, São Paulo, SP, Brasil.
} 


\section{INTRODUÇÃO}

Na busca pela redução de peso de componentes mecânicos utilizados nas indústrias automobilística e aeroespacial pode-se destacar o desenvolvimento das ligas metálicas baseadas no $\mathrm{Mg}$, que é o metal mais leve atualmente utilizado em aplicações estruturais, com massa específica de aproximadamente $1,8 \mathrm{~g} / \mathrm{cm}^{3}$ [1]. As ligas de Mg possuem boa razão entre resistência mecânica e densidade, excelente capacidade de amortecer vibrações, são recicláveis, apresentam facilidade para serem fundidas e usinadas e têm substituído com vantagem de peso o Al (densidade de aproximadamente $2,7 \mathrm{~g} / \mathrm{cm}^{3}$ ) em diversas aplicações estruturais $[1,2]$. Em contrapartida, ligas de $\mathrm{Mg}$ exibem limitações importantes tais como: baixa rigidez mecânica, baixa tenacidade e conformabilidade, baixa resistência à fluência e elevada reatividade química [3].

A baixa conformabilidade do $\mathrm{Mg}$ e de suas ligas é um fator que tem limitado a sua utilização em larga escala, posto que os produtos de processos de conformação primária como laminação e trefliação constituem matéria-prima para diversos processos secundários no setor industrial. Essa limitação é atribuída, em grande parte, à estrutura cristalina hexagonal compacta $(\mathrm{HC})$ do magnésio. Os metais de estrutura $\mathrm{HC}$ apresentam baixa capacidade de se deformarem porque possuem número limitado de sistemas de escorregamento. De fato, em baixas temperaturas, a deformação por escorregamento do $\mathrm{Mg}$ ocorre apenas nos planos basais (0001) da estrutura $\mathrm{HC}$ que se torna então incapaz de acomodar deformações extensivas, necessárias para aplicações envolvendo de conformação mecânica a frio [4, 5].

Diversos estudos apontam como solução para o problema da baixa conformabilidade do $\mathrm{Mg}$ à temperatura ambiente a redução do tamanho de grão [3-4,6]. Processos de conformação a quente efetuados com taxas lentas de deformação podem gerar microestruturas com tamanho de grão próximo de $10 \mu \mathrm{m}$ [7]. Nessas condições, a conformabilidade das ligas pode ser subsequentemente melhorada pela aplicação de deformação plástica severa, seguido de tratamento de recristalização criando estruturas de grão ultrafinas com tamanho de aproximadamente $1 \mu \mathrm{m}$ [8]. Um dos processos utilizados para refino de grão por deformação plástica à temperatura ambiente é a Extrusão em Canal Angular (ECA) [3,9]. Por exemplo, Mabuchi e colaboradores [10] conseguiram obter microestruturas com tamanho de grão de cerca de $1 \mu \mathrm{m}$ em uma liga Mg-Al-Zn. Koike e colaboradores [5] trabalharam com ligas AZ31B com tamanho médio de grão de 6,5um e observaram aumento de ductilidade das amostras pela ativação de mecanismos de deformação por maclação. Os processos de ECA, contudo, apresentam baixa produtividade [6].

Recentemente, estudos foram realizados com o objetivo de obter refino de grão de ligas de magnésio por meio da aplicação de processos de conformação convencionais como trefilação e extrusão à temperatura ambiente seguidos, em alguns casos, de tratamento de recristalização entre $250^{\circ} \mathrm{C}$ e $300^{\circ} \mathrm{C}[11,12]$. Tais processos apresentariam melhor produtividade em comparação com processos de ECA. Neste trabalho, é apresentado um estudo do processo de conformação a frio por trefilação seguido de recozimento da liga de magnésio ZK60A (Mg-6\%Zn-1\%Zr). O objetivo principal do presente estudo é descrever a cinética de recristalização da liga ZK60A o que, segundo trabalho recente, consiste em uma etapa importante no desenvolvimento estruturas de granulação fina de ligas de Mg [13]. A liga ZK60A foi selecionada devido ao efeito positivo do $Z r$ no refino do grão de $M g[14,15]$ e,

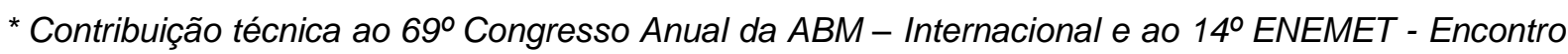
Nacional de Estudantes de Engenharia Metalúrgica, de Materiais e de Minas, 21 a 25 de julho de 2014, São Paulo, SP, Brasil.
} 
também, porque a maior parte dos trabalhos voltados ao estudo da recristalização de ligas de $\mathrm{Mg}$ foram efetuados nas ligas Mg-Zn-Al (como AZ31 ou AZ61).

\section{MATERIAIS E MÉTODOS}

\subsection{Materiais}

Barras circulares com diâmetro de 25,4 mm da liga de Mg ZK60A foram fornecidas no estado envelhecido (T5) por Metalmart International Inc (E.U.A). Antes de serem conformadas a frio, as barras foram usinadas para que apresentassem diâmetro final de $25 \mathrm{~mm}$, foram submetidas a ensaio de tração convencional e observadas no microscópio ótico. As barras recebidas no estado T5 e usinadas foram então conformadas a frio por trefilação. Os ensaios de conformação foram executados na bancada de trefilação do laboratório de conformação mecânica da Pontifícia Universidade Católica de Minas Gerais, PUC-MG [16, 17], apresentada na Figura 1. O equipamento é composto de um cilindro montado sobre uma estrutura de aço, unidade hidráulica e apoio para inserção das matrizes de trefilação (fieiras).

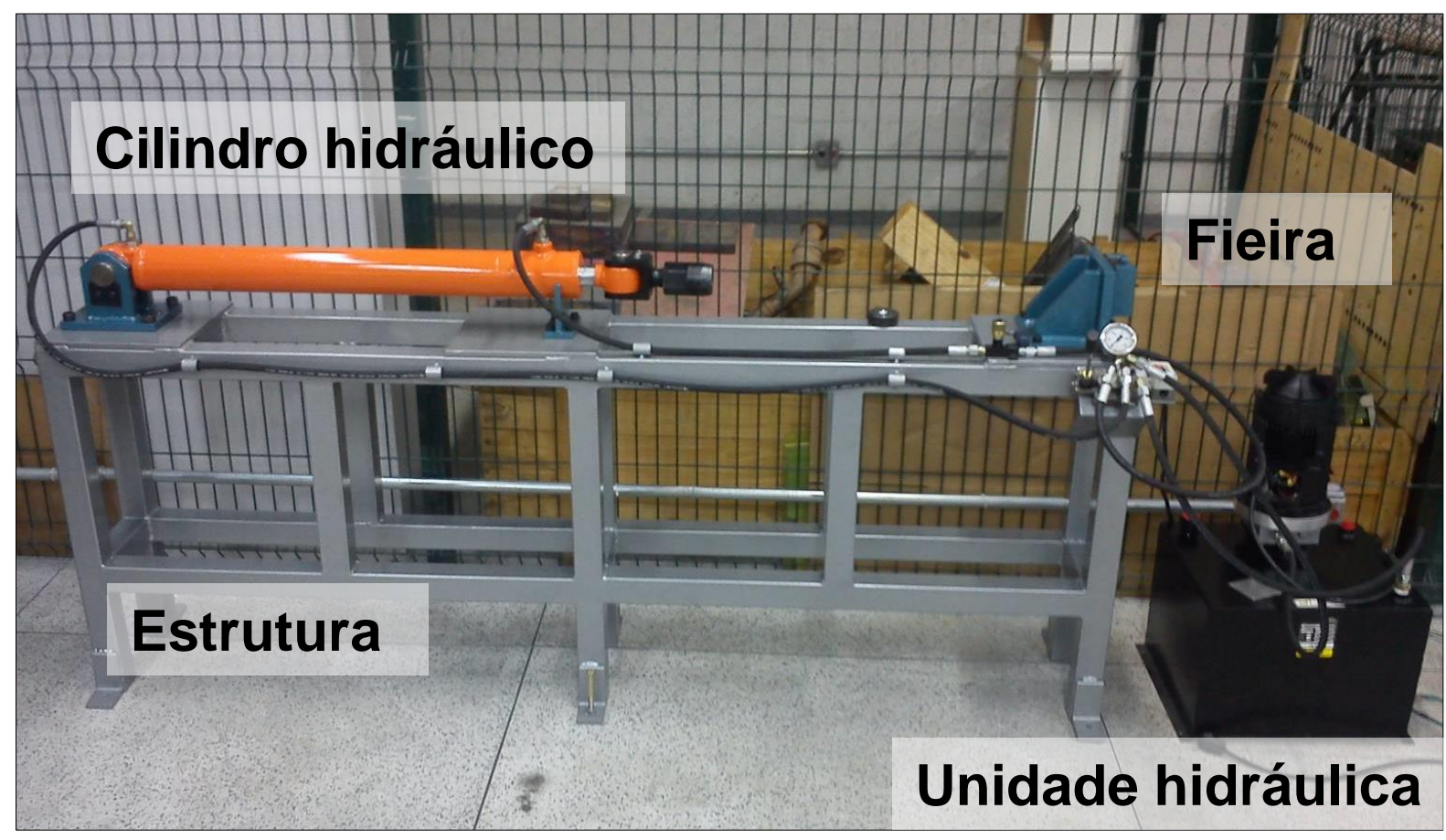

Figura 1. Bancada de trefilação do laboratório de conformação mecânica da PUC-MG [16, 17].

Foram executados diversos passes de conformação, resultando em diâmetros de 24, 23, 22 e $21 \mathrm{~mm}$, correspondentes, respectivamente, a quantidades de 7,8, 15,4, 22,6 e 29,4\% de Trabalho a Frio (\%TF). Tentativas subsequentes de redução de diâmetro levaram ao rompimento da barra durante a execução do processo de conformação. Após os ensaios de conformação, diferentes amostras dotadas do maior grau de encruamento $(29,4 \% \mathrm{TF})$ foram submetidas a recozimento de recristalização nas temperaturas de 400, 350, 300, 250, 200 e $150{ }^{\circ} \mathrm{C}$ por tempos variados. $\mathrm{O}$ aquecimento foi realizado em fornos elétricos tipo mufla, sem atmosfera controlada. O processo de recristalização foi acompanhado com a realização de ensaios de dureza Rockwell F (HRF). Para cada amostra, foram realizadas 4 medições de dureza HRF.

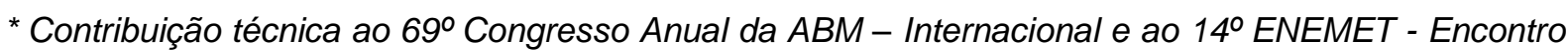
Nacional de Estudantes de Engenharia Metalúrgica, de Materiais e de Minas, 21 a 25 de julho de 2014, São Paulo, SP, Brasil. 


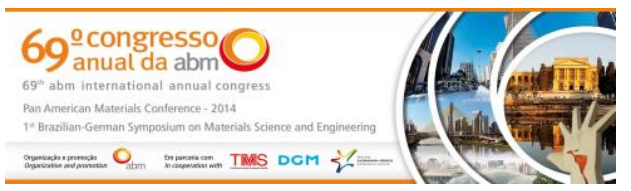

\subsection{Propriedades Mecânicas}

As propriedades mecânicas das amostras no estado T5 e nas condições $7,8,15,4$, 22,6 e 29,4\% foram avaliadas por meio de ensaios de tração. Os ensaios foram realizados em uma máquina universal de ensaios EMIC DL20000 à temperatura ambiente. Os corpos-de-prova utilizados possuíam seção reta retangular com $4 \times 10$ $\mathrm{mm}$, comprimento da área útil de $55 \mathrm{~mm}$ e foram usinados a partir das barras trefiladas, com o eixo principal orientado segundo a direção de trefilação. Os ensaios foram realizados à temperatura ambiente com uma velocidade de deslocamento de $2 \mathrm{~mm} / \mathrm{min}$ (correspondente a uma taxa de deformação de $0.04 \mathrm{~min}^{-1}$ ). Tanto as dimensões dos corpos-de-prova como as condições do ensaio obdeceram a norma ABNT NBR 6152 (2002) para ensaio de materiais metálicos.

\subsection{Microestrutura}

Amostras no estado T5 e 29,4\%TF foram preparadas para exame ao microscópio ótico. A preparação envolveu lixamento $(400,600$ e 1200) seguida de polimento fino em pasta de $\mathrm{Al}_{2} \mathrm{O}_{3}$ dispersa em álcool. Antes de serem examinadas no microscópio, as amostras foram atacadas por imersão em ácido pícrico $(5 \mathrm{ml}$ ácido acético, $6 \mathrm{~g}$ ácido pícrico, $10 \mathrm{ml}$ água e $100 \mathrm{ml}$ etanol) por $30 \mathrm{~s}$ e limpas em etanol. As imagens foram registradas em microscópio ótico Carl Zeiss Axio Lab.A1.

\section{RESULTADOS E DISCUSSÃO}

\subsection{Propriedades Mecânicas}

Os resultados dos ensaios de tração aplicados às amostras nos estados T5 e conformadas a frio são sintetizados nas Figuras 2(a) e 2(b) que apresentam, respectivamente, as curvas tensão-deformação e a evolução da razão entre o limite de escoamento (LE) e limite de resistência (LR) segundo a quantidade de trabalho a frio. A curva tensão-deformação para a amostra 15,4 \%TF não foi representada na Figura 1(a) para não prejudicar a visualização das demais linhas do gráfico.

(a)

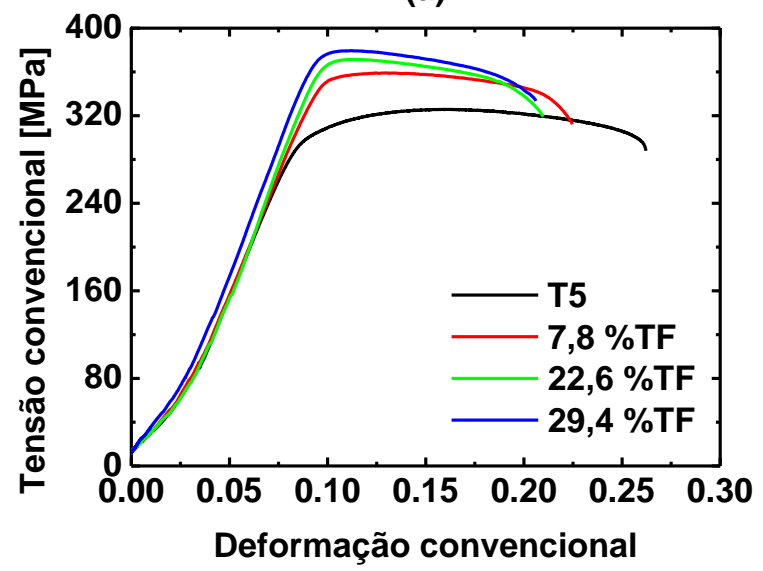

(b)

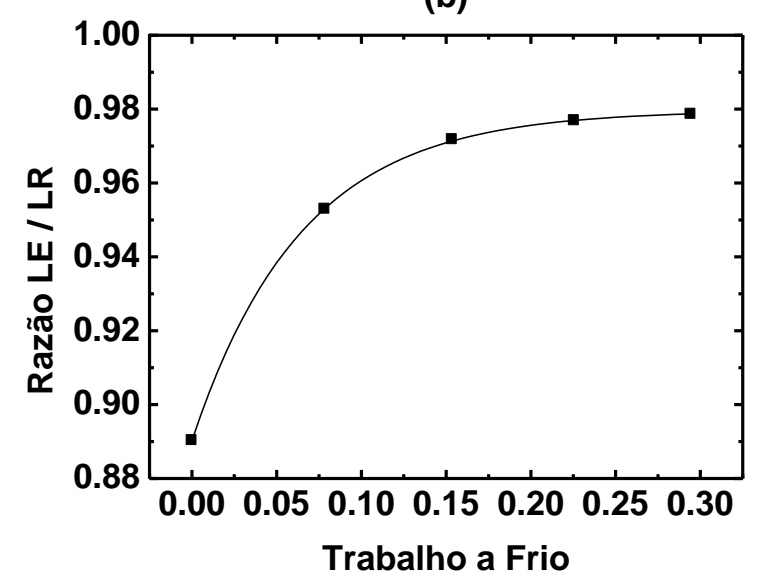

Figura 2. Resultados dos ensaios de tração: (a) curvas tensão-deformação e (b) evolução da razão entre limite de escoamento (LE) e limite de resistência (LR) segundo a quantidade de trabalho a frio.

\footnotetext{
* Contribuição técnica ao 69ำ Congresso Anual da ABM - Internacional e ao 14ํㅡㄹ ENEMET - Encontro Nacional de Estudantes de Engenharia Metalúrgica, de Materiais e de Minas, 21 a 25 de julho de 2014, São Paulo, SP, Brasil.
} 


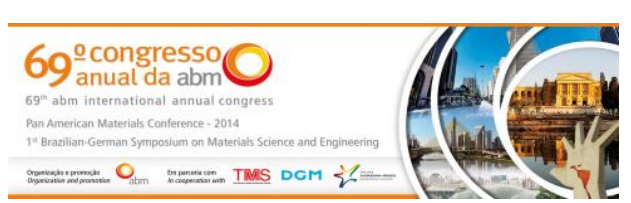

Onde y é a fração transformada, do a dureza inicial (correspondente à fração transformada nula), $d_{i}$ a dureza de um ponto para a temperatura considerada e $d_{f} a$ dureza correspondente ao total de fração transformada.

A evolução da fração transformada (y) em função do tempo de recristalização para as temperaturas consideradas é apresentada na Figura 4(b). A curva correspondente à temperatura de $400{ }^{\circ} \mathrm{C}$ de recozimento não foi representada na Figura 4(b) para possibilitar melhor visualização dos resultados. Além disso, como se pode perceber pela Figura $4(\mathrm{a})$, para a temperatura de $400{ }^{\circ} \mathrm{C}$, devido à elevada velocidade com que se deu o processo de recristalização, não foi possível realizar medições que captassem a variação de dureza entre o valor inicial de $86 \pm 0,5 \mathrm{HRF}$ e os valores que caracterizam o patamar final, o que ocorre a partir de 2 min.
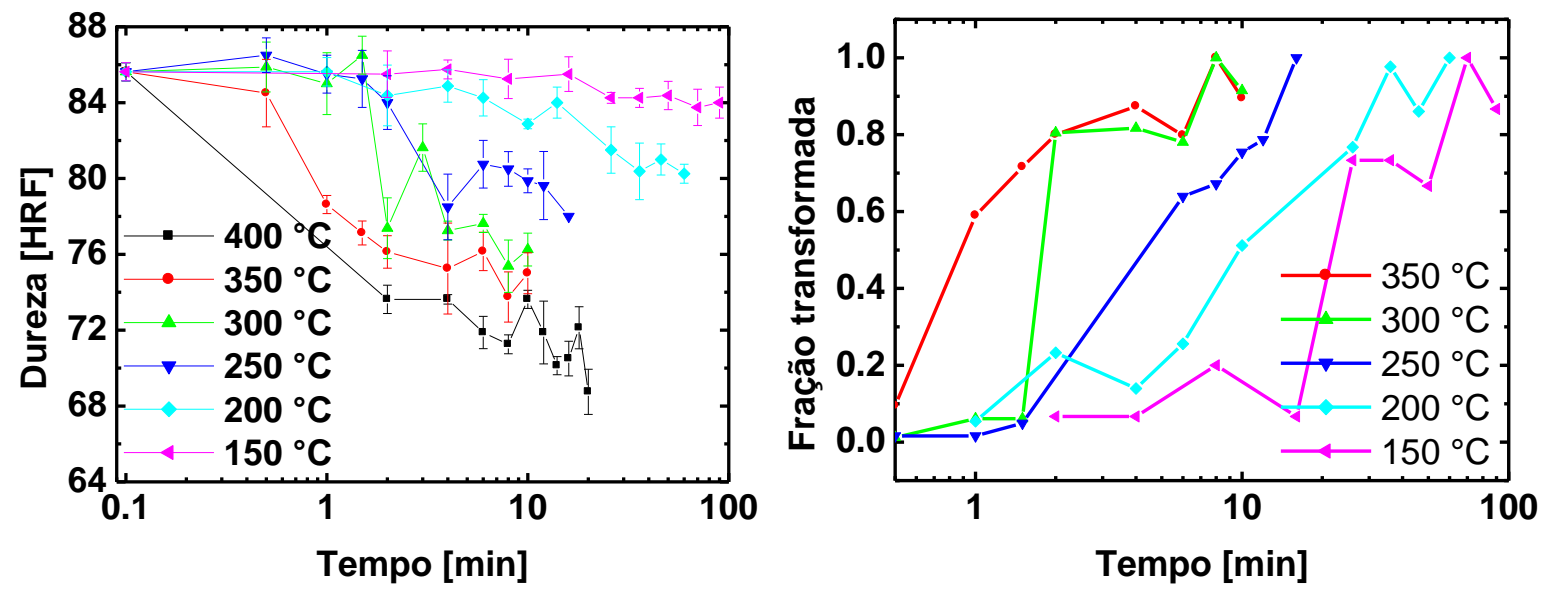

Figura 4. Acompanhamento do processo de recristalização da liga ZK60A: (a) evolução da dureza e (b) da fração transformada em função em função do tempo de recristalização.

Com base nos resultados apresentados na Figura 4, buscou-se determinar o tempo de tratamento térmico correspondente à $50 \%$ de fração transformada (t50) para cada temperatura. Os valores obtidos estão apresentados na Tabela 1.

Tabela 1. Tempos correspondentes a $50 \%$ de fração transformada $\left(t_{50}\right)$

\begin{tabular}{lc}
\hline Temperatura & $t_{50}$ \\
\hline $400^{\circ} \mathrm{C}$ & $1,4 \mathrm{~min}$ \\
\hline $350^{\circ} \mathrm{C}$ & $0,9 \min$ \\
\hline $300^{\circ} \mathrm{C}$ & $3,8 \mathrm{~min}$ \\
\hline $250^{\circ} \mathrm{C}$ & $6,2 \mathrm{~min}$ \\
\hline $200^{\circ} \mathrm{C}$ & $19,1 \mathrm{~min}$ \\
\hline $150^{\circ} \mathrm{C}$ & $37,2 \mathrm{~min}$ \\
\hline
\end{tabular}

Os resultados obtidos na Tabela 1 permitiram a determinação da energia de ativação para o processo de recristalização da liga ZK60A, por meio da aplicação da Equação 2 [4, 11]:

$$
\frac{1}{t_{50}}=A_{0} \cdot \exp \left(-\frac{Q}{R T}\right)
$$

Onde $A_{0}$ é um parâmetro independente da temperatura, $R$ é a constante universal dos gases, T é a temperatura absoluta e $Q$ a energia de ativação.

\footnotetext{
* Contribuição técnica ao $69^{\circ}$ Congresso Anual da ABM - Internacional e ao 14ํㅡㄹ ENEMET - Encontro Nacional de Estudantes de Engenharia Metalúrgica, de Materiais e de Minas, 21 a 25 de julho de 2014, São Paulo, SP, Brasil.
} 


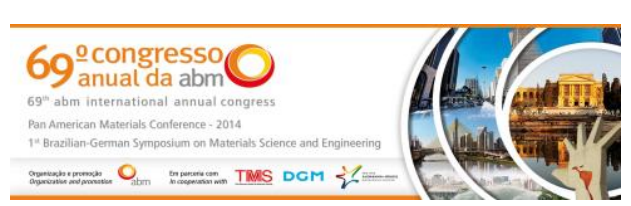

O valor da energia de ativação pôde então ser obtido a partir do coeficiente angular da reta em um gráfico relacionando o logaritmo natural do primeiro membro da Equação (2) e o inverso da temperatura absoluta. Tal procedimento resultou em um valor de $35,6 \pm 4,5 \mathrm{~kJ} / \mathrm{mol}$ para energia de ativação, conforme mostrado na Figura 5 .

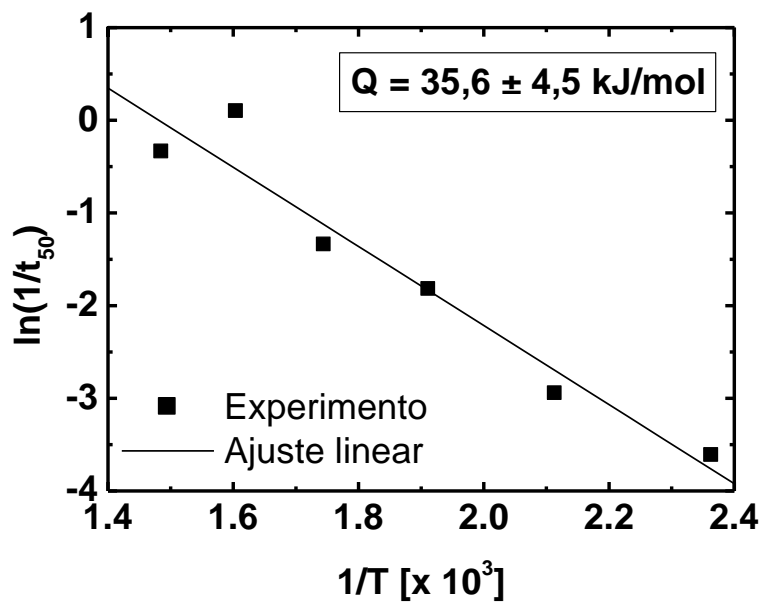

Figura 5. Determinação da energia de ativação $(Q)$ para o processo de recristalização da liga ZK60A.

Em estudo recente, Zhang et al [13] realizaram a determinação da energia de ativação para recristalização de ligas $\mathrm{Mg}-1,5 \% \mathrm{Zn}-0,6 \% \mathrm{Zr}$ contendo terras raras. Os autores encontraram valores de 23,8, 31,2, 34,7, 93,5 e 76,2 kJ/mol para concentrações de $0,0,5,1,0,2,0$ e $4,0 \%$ de terras raras. $O$ valor da energia de ativação no presente trabalho é superior a 23,8 kJ/mol, correspondente à liga $\mathrm{Mg}$ $\mathrm{Zn}-\mathrm{Zr}$ sem adição de terras raras porém próximo ao valor correspondente à liga contendo 1,0\% terras raras. Segundo Zhang et al, o aumento da energia de ativação em função de teor de terras raras se deve ao efeito à maior formação de precipitados intermetálicos ao longo dos contornos de grão da fase primária $\alpha-M g$ e ao enriquecimento de elementos de terras raras em solução sólida na fase $\alpha-M g$. Com base nessas informações, se pode inferir que o maior teor de $\mathrm{Zn}$ presente na liga ZK60A levou ao aumento da fração de compostos intermetálicos distribuídos ao longo dos contornos de grão da fase $\alpha-\mathrm{Mg}$, limitando a mobilidade dos átomos e, consequentemente, aumentando a energia de ativação para a recristalização. Para melhor explorar essa questão, uma análise detalhada com auxílio de microscopia eletrônica da evolução da microestrutura da liga ZK60A durante o processo de recristalização se faria necessária.

\section{CONCLUSÃO}

No presente trabalho o processo de trefilação a frio foi aplicado a barras circulares da liga de magnésio ZK60A recebida incialmente na condição T5. Foram realizados passes sequenciais de conformação para geração de quantidades de trabalho a frio de $7,8,15,4,22,6$ e 29,4\%. As amostras apresentaram tendência ao encruamento após cada passe de trefilação, o que se evidenciou pelo aumento da resistência, perda de ductilidade aproximação dos valores de limite de escoamento e limite de resistência. Após os ensaios de conformação, as amostras com 29,4 \%TF foram submetidas a tratamento térmico de recristalização às temperaturas de 400,350 , 300, 250, 200 e $150{ }^{\circ} \mathrm{C}$. Nas condições apresentadas, determinou-se a energia de

\footnotetext{
* Contribuição técnica ao $69^{\circ}$ Congresso Anual da ABM - Internacional e ao 14ํㅡㄹ ENEMET - Encontro Nacional de Estudantes de Engenharia Metalúrgica, de Materiais e de Minas, 21 a 25 de julho de 2014, São Paulo, SP, Brasil.
} 


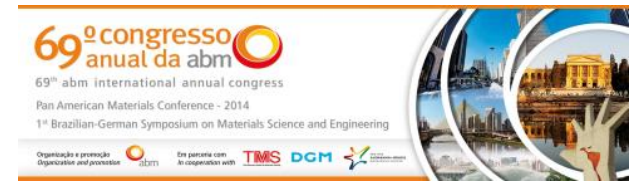

ativação para o processo de recristalização da liga ZK60A e o valor encontrado foi de $35,6 \pm 4,5 \mathrm{~kJ} / \mathrm{mol}$.

\section{Agradecimentos}

O presente trabalho foi financiado pela Pontifícia Universidade Católica de Minas Gerais por meio do Fundo de Incentivo à Pesquisa (FIP), com número de projeto 2013/7626-S1.

\section{REFERÊNCIAS}

1 Froes FH, Eliezer D, Aghion E, The Science, Technology and Applications of Magnesium. Journal of the Minerals, Metals and Materials Society. 1998; 50(9): 30-34.

2 Lima DRS. Desenvolvimento e Controle de Processos de Conformação da Liga de Magnésio AZ61. Tese (Doutorado). Porto Alegre: Universidade Federal do Rio Grande do Sul; 2012.

3 Mordike BL, Ebert T. Magnesium - Properties - Applications - Potential. Materials Science \& Engineering A. 2001; 302: 47-45.

4 Reed-Hill RE. Physical Metallurgy Principles. 2 $2^{\underline{a}}$ ed. Monterey: Litton Educational Publishing; 1973.

5 Koike J, Kobayashi T, Mukai T, Watanabe H, Suzuki M, Maruyama K, Higashi K. The activity of non-basal slip systems and dynamics recovery at room temperature in finegrained AZ31B magnesium alloys. Acta Materialia. 2003; 51: 2055-2065.

6 Del Valle JA, Pérez-Prado MT, Ruano OA. Texture evolution during large-strain hot rolling of the Mg AZ61 alloy. Materials Science \& Engineering A. 2003; 355: 68-78.

7 Chao $\mathrm{H}$, Wang E. Achieving ultrafine grain size in Mg-Al-Zn alloy by cold drawing. International Journal of Modern Physics B. 2009; 23(6-7): 927-933.

8 Sakai T, Miura H. Mechanical Properties of Fine-Grained Magnesium Alloys Processed by Severe Plastic Forging, in: Czerwinski F. Magnesium Alloys - Design, Processing and Properties, InTech; 2011.

9 Chang TC, Wang JY, O CM, Lee, S. Grain refining of magnesium alloy AZ31 by rolling. Journal of Materials Processing Technology. 2003; 140: 588-591.

10 Mabuchi M, Iwasaki H, Yanase K, Higashi K, Low temperature superplasticity in an AZ91 magnesium alloy processed by ECAE. Scripta Materialia. 1997; 36(6): 681-686.

11 Chao HY, Sun HF, Chen WZ, Wang ED, Static recrystallization kinetics of a heavily cold drawn AZ31 magnesium alloy under annealing treatment. Materials Characterization. 2011; 62: 312-320.

12 Liang SJ, Liu ZY, Wang ED. Microstructure and mechanical properties of Mg-Al-Zn alloy deformed by cold extrusion. Materials Letters. 2008; 62: 3051-3054.

13 Zhang J, Li W, Guo Z, Static recrystallization and grain growth during annealing of an extruded Mg-Zn-Zr-Er magnesium alloy. Journal of Magnesium and Alloys. 2013; 1: 3138.

14 Luo A, Pekguleryuz MO, Cast magnesium alloys for elevated temperature applications. Journal of Materials Science. 1994; 29: 5259-5271.

15 Lee YC, Dahle, AK, St. John DH. The role of solute in grain refining magnesium. Metallurgical and Materials Transactions A. 2000; 31: 2895-2906.

16 Silva EV, Souza, RAA, Librelon, T. Projeto Bancada de Trefilação: Desenvolvimento Sistema Hidráulico. Trabalho de Conclusão de Curso (Graduação). Contagem: Pontifícia Universidade Católica de Minas Gerais; 2011.

17 Correa BF, Murta RMA. Projeto Bancada de Trefilação: Desenvolvimento Estrutural. Trabalho de Conclusão de Curso (Graduação). Contagem: Pontifícia Universidade Católica de Minas Gerais; 2011.

\footnotetext{
* Contribuição técnica ao $69^{\circ}$ Congresso Anual da ABM - Internacional e ao 14ํㅡㄹ ENEMET - Encontro Nacional de Estudantes de Engenharia Metalúrgica, de Materiais e de Minas, 21 a 25 de julho de 2014, São Paulo, SP, Brasil.
} 